\title{
Une possibilité de prise en considération de critères acoustiques pour le calcul aérodymamique d'un ventillateur axial
}

\section{A possibility of taking acoustic criteria into consideration in the aerodynamic calculation for an axial fan}

\author{
F. Bario \\ Docteur de troisième cycle \\ ingénieur \\ Société METRAFLU*
}

\section{Introduction}

La mise en vigueur récente de normes et de réglementations de plus en plus sévères en matière de lutte contre le bruit, associée à une sensibilisation du public aux problèmes de nuisances font qu'aujourd'hui, la qualité de discrétion acoustique d'une installation est un des facteurs importants intervenant lors du choix du matériel. Dans le domaine des turbomachines en particulier, on comprendrait mal le choix d'un acheteur se portant acquéreur d'un ventilateur qui, à toutes performances égales et à prix équivalents, serait plus bruyant qu'un matériel présenté par une firme concurrente. Le problème du bruit se pose donc sur un plan purement écologique, mais également sur un plan économique.

Dès que l'on a besoin de débits importants associés à de faibles taux de pression, le ventilateur axial est nettement plus adapté que le ventilateur centrifuge, que ce soit du point de vue performances ou du point de vue coût de fabrication. Cependant, à performances aérauliques égales, le niveau de puissance sonore est supérieur de 10 à $20 \mathrm{~dB}$ avec un ventilateur axial. Un effort important doit donc être foumi pour diminuer le bruit de ce type de machine.

Dans le cadre d'un contrat D.G.R.S.T. [1], les Sociétés Metraflu et Neu ont développé conjointement une étude de ventilateur axial "silencieux". Une étude théorique du bruit d'origine aérodynamique émis par un ventilateur a été effectuée. Il a été décidé de construire une machine axiale prototype en introduisant à la conception même de cette machine, une optimisation des paramètres responsables de l'émission de ce bruit. L'étude aérodynamique a été réalisée par le groupe Turbomachines de l'Ecole Centrale de Lyon.

Nous allons présenter dans ce papier, la façon dont a été mené le dessin aérodynamique du ventilateur axial et comment y ont été introduits un certain nombre de critères de réduction du bruit d'origine aérodynamique.

\footnotetext{
* 24 bis chemin des Mouilles 69130 Ecully
}

\section{Méthode de dessin d'une turbomachine axiale}

Pour des machines axiales ou quasi-axiales, l'écoulement tridimensionnel est le plus souvent déterminé par des méthodes simplifiées. Dans ce type de méthodes que nous avons utilisé, l'écoulement est remplacé par la combinaison d'un écoulement dans le plan méridien et d'un écoulement dans le plan circonférenciel, c'est-à-dire par deux écoulements bidimensionnels.

Les hypothèses suivantes peuvent être utilisées : - les roues (rotor-stator) sont assimilées à des surfaces de discontinuité,

- l'écoulement est supposé stationnaire et axisymétrique,

- la déflexion tangentielle de l'écoulement est donnée par la spécification de l'échange d'énergie désiré,

- l'écoulement est déterminé en des sections hors des roues,

- Les gradients d'énergie et d'entropie peuvent être pris en considération dans les conditions amont,

- l'écoulement est supposé adiabatique.

Les conditions d'équilibre appliquées au fluide permettent d'établir les composantes méridiennes qui sont associées aux composantes périphériques de vitesse.

Les triangles de vitesses ainsi obtenus, servent à établir les profils des aubages qui produiront la déflexion désirée de l'écoulement.

\subsection{Conditions d'équilibre}

Les équations du mouvement s'écrivent [2]

avec

$$
\begin{gathered}
\vec{\nabla} H=\vec{V} \times(\vec{\nabla} \times \vec{V})+T \vec{\nabla} S \\
\vec{\nabla}=\vec{i} \frac{\partial}{R \partial \theta}+\vec{j} \frac{\partial}{\partial z}+\vec{k} \frac{\partial}{\partial R}
\end{gathered}
$$

(où $H$ est l'enthalpie, $S$ l'entropie, $T$ la température, $\theta$ la direction circonférencielle, $z$ la direction axiale, $R$ la direction radiale ou le rayon, $V$ la vitesse absolue). 
Ces équations, développées dans un repère cylindrique en tenant compte des hypothèses exposées précédemment conduisent à :

$$
\frac{d V_{a}^{2}}{d R}+F V_{a}^{2}+G=0
$$

avec

$$
\begin{aligned}
& F=-\frac{2}{V a} \frac{\partial V r}{\partial z}-\frac{1}{C p} \frac{1}{\cos ^{2} \lambda} \frac{\partial S}{\partial R} \\
& G=2 \frac{V u}{R} \frac{\partial(R V u)}{\partial R}-2 \frac{\partial H}{\partial R}+\frac{1}{C p}\left(2 H-V u^{2}\right) \frac{\partial S}{\partial R}
\end{aligned}
$$

et avec $V a$ : vitesse axiale, $V u$ : vitesse tangentielle, $V r$ : vitesse radiale, $C p$ : chaleur spécifique, $\lambda$ : angle de la ligne de courant et de la direction axiale.

Nous voyons donc que cette équation régit la distribution de la vitesse axiale suivant le rayon de la machine.

\subsection{Méthode de résolution}

La solution de l'équation ci-dessus doit être trouvée par approximations successives car nous sommes obligés. de supposer une série de surfaces de courant qui doivent en plus satisfaire l'équation de continuité. L'écoulement à l'entrée de l'étage (amont roue mobile) doit être connu. Il faut alors déterminer les distributions de vitesse dans les plans aval roue mobile et aval redresseur pour des distributions de vitesses périphériques données à l'amont de l'étage et pour un échange d'énergie fixé dans la roue mobile. La solution de l'équation est du type

$$
V a^{2}=\exp \left(-\int F d R\right)\left[C-\int G \exp \left(\int F d R\right) d R\right]
$$

où $C$ est une constante à déterminer pour satisfaire le débit.

\section{Bruit émis par une machine tournante axiale [3] [4]}

\subsection{Bruit émis par un rotor}

Un rotor émet du bruit à des fréquences discrètes $m B \Omega$, multiples $m$ de la vitesse de rotation angulaire $\Omega$ du rotor et de son nombre de pales $B$. Ce bruit est dû à la mise en rotation des forces s'exerçant sur les aubages du rotor.

Les composantes du bruit émis par les forces stationnaires ont pour expression (fig. 1) :

$$
C m B=\frac{(i)^{-m B+1}}{2 \pi a_{0} r_{1}} m B \Omega\left\{\frac{x T_{R}}{r_{1}}-\frac{D_{R}}{M}\right\} \operatorname{Jn} B\left(m B \frac{M y}{r_{1}}\right)
$$

facteur de directivité

dans laquelle

$\begin{array}{cl}J m B & \text { est une fonction de Bessel } \\ M & \text { un nombre de Mach } \\ a_{0} & \text { la célérité du son } \\ x, y, r_{1} & \text { des paramètres définis fig. } 1\end{array}$

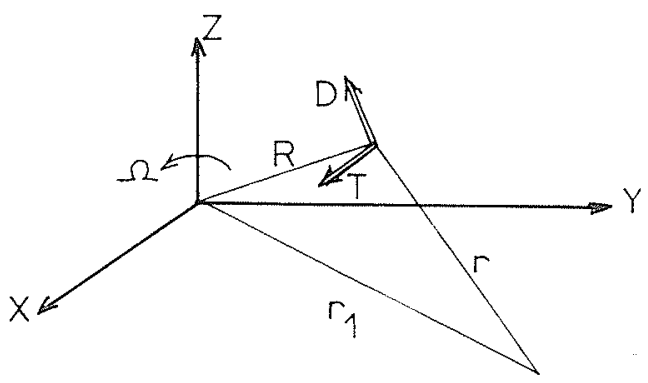

Figure 1

L'intensité acoustique rayonnée est alors:

$$
I=\sum_{m}|C m B|^{2}
$$

Le bruit du rotor est donc essentiellement lié à la vitesse de rotation $\Omega$ et aux efforts $T$ axial, $D$ circonférentiel sur la s'exerçant sur les aubes ( $R$ indice rotor).

\subsection{Bruit émis par un stator précédé d'un rotor}

L'amplitude des composantes de bruit émis à la fréquence $m B \Omega$ a pour expression

$$
\begin{aligned}
C m B=\sum_{k=\infty}^{+\infty}-\frac{i m B V \Omega}{2 \pi a_{0} r_{1}}\left\{\frac{x T_{s}}{r_{1}}-\frac{m B-k V}{m B V} D_{s}\right. & \\
J_{m B-k V} & \left(\frac{m B M y}{r_{1}}\right)
\end{aligned}
$$

On note que la fréquence d'émission est imposée par la vitesse de rotation $\Omega$ du rotor, les efforts $T_{S}$ et $D_{S}$ sont les harmoniques de la charge de pale du stator qui se déduisent des composantes stationnaires par une loi de décroissance en $(m B \Omega)^{-2}, V$ étant le nombre de pales du stator.

\section{Optimisation du bruit émis par une machine tournante axiale}

On sait que le bruit émis par un ventilateur axial varie à la puissance 6 de la vitesse de rotation de la machine. Il faut cependant se méfier de l'idée selon laquelle il suffirait seulement de diminuer la vitesse d'entraînement pour réduire le bruit. Cette idée est évidemment vraie lorsqu'un ventilateur existant est utilisé à différentes vitesses de rotation. Cependant, lorsque des spécifications de débit masse $m_{s}$ et d'élévation de pression d'arrêt $\Delta P_{T}$ sont requises, spécifications typiques imposées lors d'un projet, il convient d'analyser l'influence du paramètre vitesse d'entraînement $\Omega$.

\subsection{Contribution de la force tangentielle $D_{R}$ station- naire à l'émission du bruit}

Lorsque le débit massique $m_{s}$ et l'élévation d'enthalpie $\Delta H$ dans l'étage sont fixées, la puissance $P_{u}=$ $m_{s} \Delta H$ de la machine est donc fixée. Cette puissance s'écrit également :

$$
P_{u}=m_{s} \Delta H=\int_{R} D_{R} \Omega d R
$$


ce qui implique que le terme $\Omega D_{R}$ de la formulation des composants du bruit soit constant. La diminution de la vitesse de rotation $\Omega$ ne va donc pas contribuer à réduire la composante de bruit due à la torce station. naire $D_{R}$.

\subsection{Contribution de la force axiale stationnaire $T_{R} \grave{a}$ l'émission du bruit}

La force axiale $T_{R}$ est directement liée au saut de pression statique dans la roue mobile. Il est donc possible de réduire cette force axiale en diminuant le saut de pression statique dans la roue, c'est-à-dire en effectuant de fortes déflexions associées à une faible vitesse de rotation. Le stator ayant alors une charge plus importante.

La diminution de la vitesse de rotation $\Omega$ va donc contribuer à affaiblir la composante de bruit due à la force stationnaire $T_{R}$ du rotor.

\subsection{Contribution des forces instationnaires}

La génération acoustique est principalement due au caractère fluctuant (ou instationnaire) des forces aéro. dynamiques s'exerçant sur les aubages.

Une étude effectuée par Bridelance [5] a montré que la force $T$ instationnaire était la principale responsable du bruit émis. Si l'on considère un aspect quasistationnaire du problème, on peut relier cette force instationnaire à une force stationnaire. La force $T$ étant liée directement un saut de pression statique $\Delta P_{S}$, on peut écrire :

$$
T \sim \Delta P_{S}
$$

D'autre part, nous avons à caractériser le groupement $\Omega T$ : Le bruit sera proportionnel à $\Omega T \sim U \Delta P_{S}$. $\mathrm{La}$ vitesse de rotation $U$ est liée au saut de pression d'arrêt par la relation $\Delta P_{T}=\rho U \Delta V_{u}$ (où $\rho$ est la masse volumique) d'où la proportionalité du bruit émis avec le groupement $\Delta P_{S} \Delta P_{T} / \Delta V_{u}$. Pour le cas d'un ventilateur axial avec conditions d'entrée et de sortie identiques (pas de rotation de l'écoulement), le saut de pression statique et le saut de pression d'arrêt sont confondus. Les spécifications $\Delta P_{T}, m_{s}$ étant données, le groupement $\Delta P_{S} \Delta P_{T}$ l $\Delta V_{u}$ est inversement proportionnel à $\Delta V_{u}$. Il convient donc pour diminuer le bruit émis de réduire la vitesse d'entraînement et de charger au maximum les aubages.

\subsection{Effets de pertes}

En général, on constate que le minimum de puissance acoustique émise est obtenu pour un ventilateur axial lorsque celui-ci fonctionne à son point de rendement maximal, c'est-à-dire avec un écoulement le mieux adapté du point de vue aérodynamique. Ceci est en accord avec les hypothèses d'une émission de bruit reliée aux caractéristiques turbulentes des couches visqueuses des aubages.

\section{Caractéristiques géométriques et aérauliques du ventilateur}

Les caractéristiques géométriques et aérauliques imposées pour ce ventilateur sont les suivantes:
- rapport de moyeu $\nu=R$ moyeu $_{/} R$ carter $\geqslant 0.6$

- diamètre extérieur $2 R_{\text {carter }}=0.708 \mathrm{~m}$

Pour des raisons de recherche d'un prix de revient le plus bas possible, la veine a été définie sans directrices d'entrée (prédistributeur) et de forme cylindrique.

Les caractéristiques de pression et de débit sont les suivantes :

- débit $m_{s} / \rho=7.05 \mathrm{~m}^{3} / \mathrm{s}$

- élévation de pression d'arrêt $\Delta P_{T}=750 \mathrm{~Pa}$

Le choix de la distribution de l'échange d'énergie en fonction du rayon a été effectué de manière à obtenir en aval de la roue mobile, une vitesse axiale constante suivant le rayon. L'écoulement à l'aval de l'étage devra être axial.

On notera que de telles spécifications ne laissent de liberté qu'au niveau du choix de la vitesse de rotation et du coefficient de débit $\dot{\phi}=m_{s} / A \Omega R_{\text {carter }}$ (où $A$ est la surface interne du conduit)

\section{Optimisation acoustique et aérodynamique du ventilateur}

En fonction de l'analyse développée précédemment, les hypothèses d'optimisation acoustique du dessin de la machine sont les suivantes:

- les zones fortement perturbées aérodynamiquement sont génératrices de bruit,

- la diminution de la vitesse d'entraînement conduit à une diminution du bruit,

- les contributions du rotor et du stator au bruit global sont supposées équivalentes,

- les spectres de raies à fréquences discrètes observés pour des rotors axiaux sont créés par les forces quasistationnaires.

Nous avons donc admis les principes directeurs suivants :

- réalisation d'un dessin aérodynamique optimisé de façon à éviter les décollements sur les aubages,

- recherche d'une solution à faible vitesse d'entraînement conduisant à un étage aérodynamiquement très chargé,

- recherche d'une minimisation des vitesses relatives maximales présentes dans l'étage.

- réalisation du dessin géométrique des roues en utilisant les informations concernant les nombres optimaux de pales du rotor et du stator [1].

\section{Méthode de calcul}

Plusieurs calculs du champ aérodynamique ont été effectués en faisant varier la vitesse de rotation $\Omega$ et le rapport de moyeu $\nu$. Ces calculs d'équilibre radial sont effectués aisément. Ils se réduisent à des déterminations de triangles de vitesses étant donné que dans un premier temps ni pertes ni courbures ne sont introduites. Les évolutions des vitesses relatives maximales pour le rotor $W_{1 \text { carter }}$ (vitesse relative en tête de pale à l'amont du rotor) et pour le stator $V_{2}$ moyeu (vitesse absolue en pied de pale à l'amont du stator) sont présentées, figure 2 . 


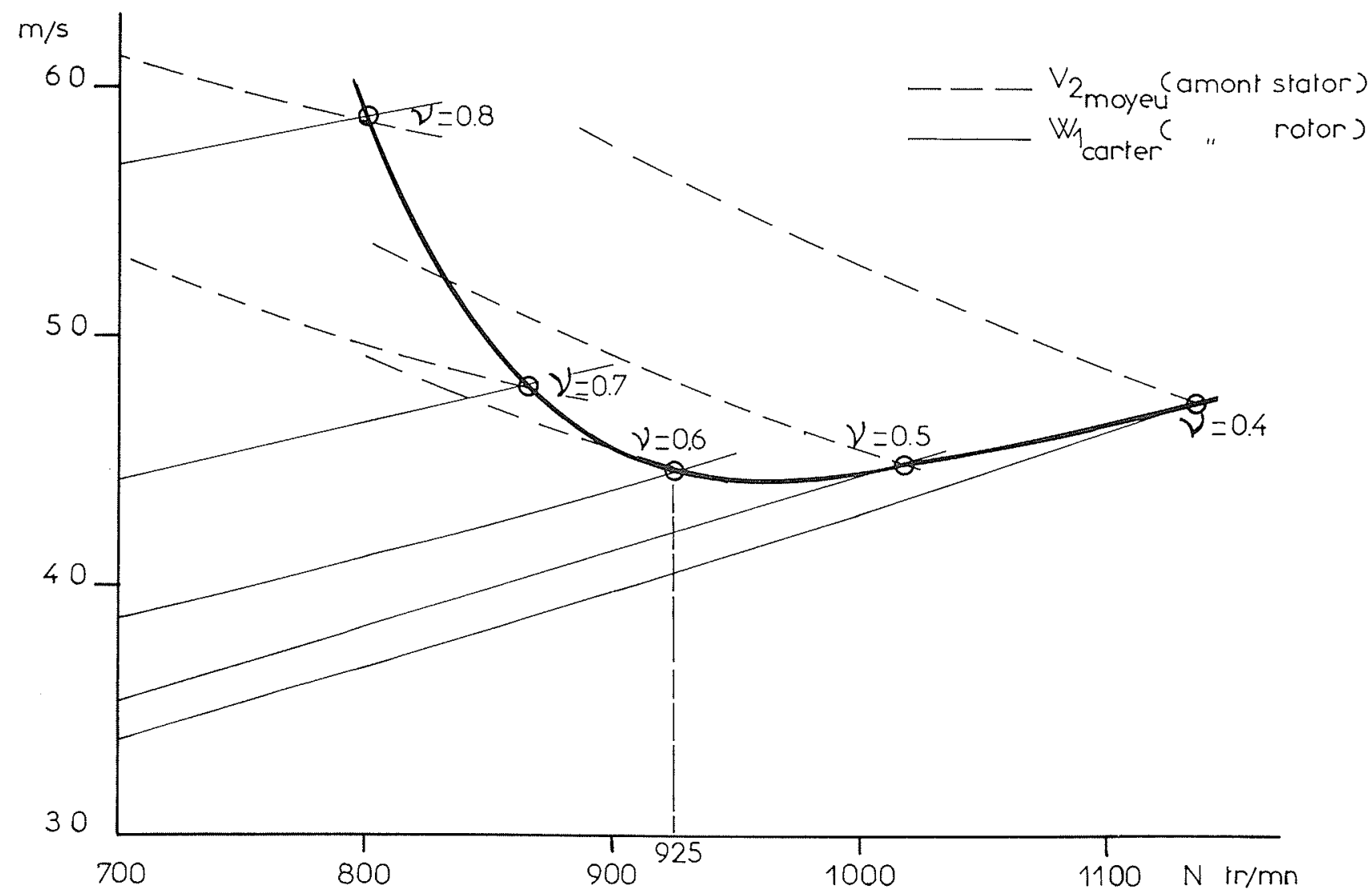

Figure 2 - Evolution des vitesses relatives maximales pour le rotor et le stator.

Les rapports de moyeu $\nu=0,4$ et $\nu=0,5$ correspondent à des conditions de fonctionnement de l'étage irréalistes (rapport de vitesses relatives en pied supérieur à 1 ; stator trop chargé). Le rapport de moyeu $\nu=0,6$ a été considéré optimum, le maximum de vitesse dans l'étage est alors de $45 \mathrm{~m} / \mathrm{s}$ pour une vitesse de rotation de $925 \mathrm{tr} / \mathrm{min}$.

Un calcul complet de l'écoulement a été ensuite réalisé au moyen d'une méthode de résolution des équations. Cette méthode prend en considération les pertes de profils et les courbures.

Les aubages sont alors calculés à l'aide des triangles de vitesse obtenus par utilisation des corrélations Naca [6]. Le nombre d'aubages choisi est de 12 pour le rotor et de 17 pour le stator, cette combinaison, dêjà connue par les établissements Neu pour son efficacité acous- tique a été étudiée par la Société Metraflu. Un calcul théorique a permis de mettre en évidence cette efficacité [1].

\section{Résultats expérimentaux obtenus}

Le ventilateur calculé a été fabriqué et expérimenté par la Société Neu. Il est présenté figures 3 et 4.

Les caractéristiques débit-pression sont comparés à celles données par un ventilateur axial de référence présentant les mêmes caractéristiques géométriques et le même nombre d'aubages, mais tournant à une vitesse de rotation $N$ de $1420 \mathrm{tr} / \mathrm{min}$. Le ventilateur dessiné a été essayé à $960 \mathrm{tr} / \mathrm{min}$. Les mesures de bruit sont effectuées face à l'aspiration du ventilateur. Les

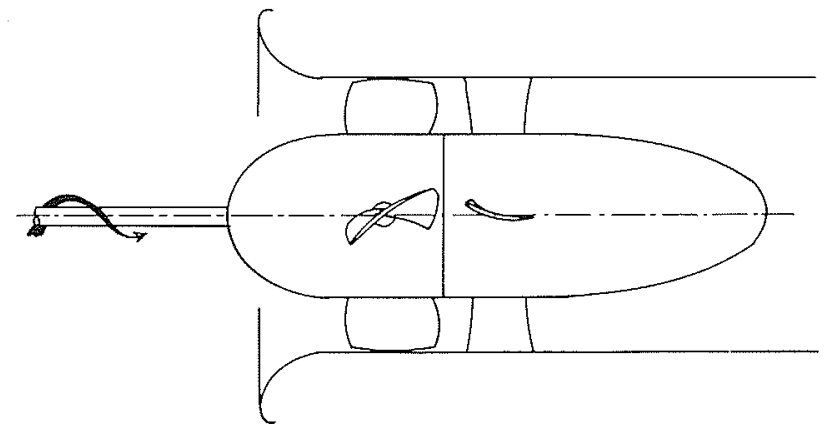

Figure 3 - Le ventilateur.

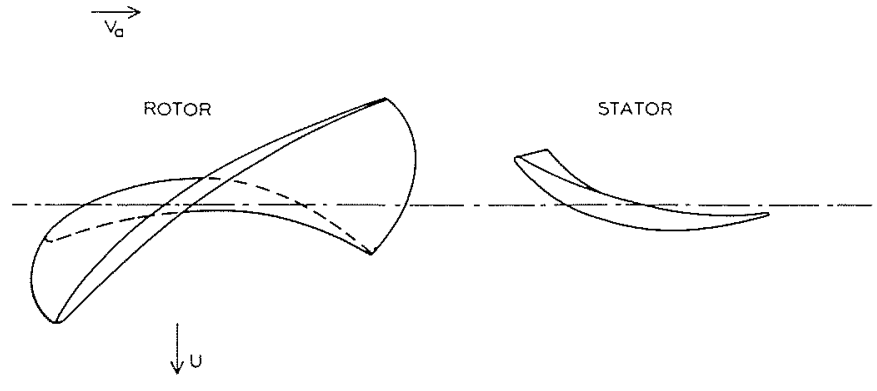

Figure 4 - Profils des aubages. 


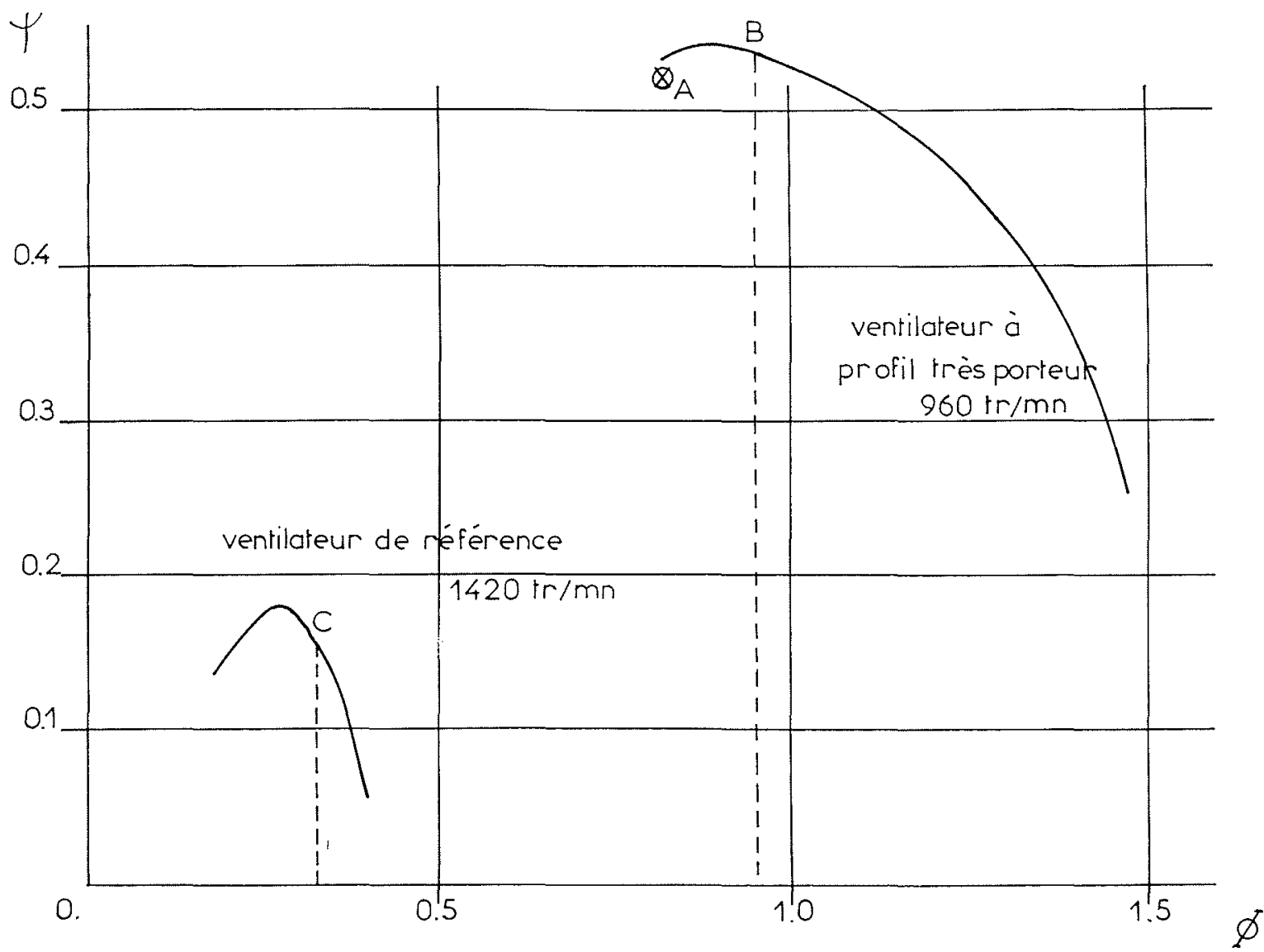

Figure 5 - Courbes de performances comparées.

performances comparées sont présentées, figure 5, sous forme d'un cofficient de pression

$$
\psi=\Delta P_{T} / \rho\left(\Omega R_{\text {carter }}\right)^{2}
$$

et d'un cœfficient de débit

$$
\phi=m_{s} / \rho R_{\text {carter }}^{3}\left(1-\nu^{2}\right) \pi \Omega
$$

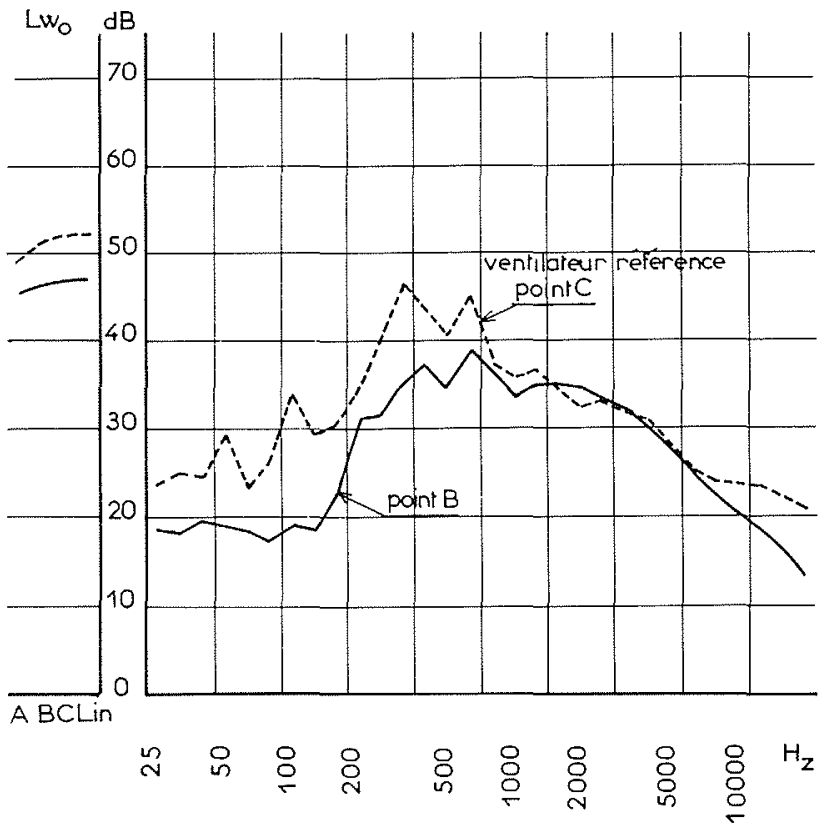

Figure 6 - Spectres de bruit comparés.
Le ventilateur dessiné donne à isovitesse d'entraînement, trois fois plus de débit et de pression que le ventilateur de référence.

Le point de calcul $A$ est légèrement au-dessous de la courbe expérimentale (point de calcul : $m_{s} / \rho=7.32 \mathrm{~m}^{3} / \mathrm{s}$, $\Delta P_{T}=810 P a$ à $960 \mathrm{tr} / \mathrm{min}$ ). Le rendement maximal obtenu est $\eta$ maxi $=0.86$ au point $B$, alors que le rendement maximal du ventilateur de référence est $\eta=0.85$.

Les niveaux de puissance sonore spécifique $L \omega_{0}$ des deux ventilateurs sont comparés en prenant comme caractéristiques de base $\left(m_{s} / \rho\right)_{0}=1 \mathrm{~m}^{3} / \mathrm{s}$ et $\Delta P_{0}=10 P a$. Le $L \omega$ linéaire du ventilateur de référence à son meilleur rendement est $L \omega_{0}=52 \mathrm{~dB}$; celui du ventilateur dessiné est $L \omega_{0}=47 \mathrm{~dB}$.

A égalité de caractéristiques débit-pression et en supposant travailler au rendement optimal, le $L \omega_{0}$ linéaire du ventilateur, à profil très porteur, est inférieur de $5 d B$ à celui du ventilateur classique de référence de cette étude. Le spectre de bruit du ventilateur très porteur à l'avantage de présenter un niveau plus faible dans les basses fréquences situées à gauche du fondamental (192 Hz) (fig. 6).

\section{Conclusions}

Le calcul aérodynamique d'un ventilateur axial, effectué en introduisant des critères acoustiques simples, a conduit à une amélioration substancielle du niveau de bruit. Un gain de $5 d B$ Lin sur le niveau de 
puissance sonore spécifique a été obtenu, le ventilateur utilisé comme référence étant déjà reconnu peu bruyant en niveau sonore spécifique. De plus, les niveaux basses fréquences ont été réduits de façon sensible.

Il apparaît donc que l'atténuation du bruit d'un venti. lateur axial peut être réalisée, à la conception même de la machine, en appliquant les règles simples suivantes :

- recherche d'une charge aérodynamique maximale compatible avec des couches limites non décollées sur les aubages, associée à une vitesse d'entraînementminimale,

- recherche d'une minimisation des vitesses relatives maximales dans l'étage,

- utilisation de l'information concernant les nombres de pales du rotor et du stator.

\section{Bibliographie}

[1] D.G.R.S.T., METRAFLU, NEU - Bruit de ventilateurs axiaux équipés de profils très proteurs. Contrat D.G.R.S.T. $\mathrm{n}^{\circ} 76.7 .1018$ et 76.7 .1019 (1976).

[2] VAVRA - Aerothermodynamics and flow in turbomachines. R.E. Kieger Publishing Co. New York (1974).

[3] LOWSON M.V. - The sound field for singularities in motion. Proc. Roy. Soc., - A286, 559-572 (1965).

[4] ARBEY H. - Ecole Centrale de Lyon. Communications privées.

[5] BRIDELANCE J.P. -- Analyse du bruit des ventilateurs axiaux à l'aide du formalisme de Lowson. $7^{\mathrm{e}}$ Colloque d'Acoustique Aéronautique - Lyon - 4 et 5 Novembre 1980.

[6] Aerodynamic design of axial flow compressors. NASA SP36, Lewis Research Center, Cleveland (1965).

\section{Discussion}

Président : R. CANAVELIS

M. le Président. - Je remercie M. BARIO. Y a-t-il des questions de la part de l'auditoire?

M. BOVIS. - Vous avez fait une étude acoustique qui repose sur les lois du rayonnement du rotor et du stator. Comment prenez-vous en compte le conduit qui se trouve autour de vos éléments tournants ou fixes, non seulement pour des questions d'interaction mécanique, mais aussi pour des questions de propagation?

M. BARIO. - Dans le calcul que vous avez vu, il y a une formulation acoustique simple : c'est la formule de Lowson qui est dérivée de l'étude acoustique. Elle nous a permis de définir uniquement des critères qui sont liés à la roue, donc le conduit par lui-même n'est pas inclus. On travaille uniquement sur la roue et le stator.

Les mesures de bruit dans les deux cas, avec ventilateur de référence, ont été faites dans les mêmes conditions, donc on peut supposer que les phénomènes liés au conduit sont les mêmes dans les deux cas.

M. LEFEBVRE. - Vous avez fait un essai à $960 \mathrm{tr} / \mathrm{min}$. pour le ventilateur et un autre à $1.400 \mathrm{tr} / \mathrm{min}$. pour le ventilateur de référence. Il aurait été intéressant d'avoir une variable de vitesse.

M. BARIO. - Les essais présentés effectués par la Société NEU n'ont pas eu lieu à des vitesses différentes.

Par contre, les bruits ont été relevés en différents points de fonctionnement.

$M$. le Président. - Il semblerait, d'après M. BARIO, que d'une vitesse à l'autre on puisse transposer en $V^{6}$.

M. BARIO. - Je pense que les acousticiens utilisent des formules empiriques qui permettent de comparer les performances différentes des deux ventilateurs.
M. le Président. - Est-ce que l'ordre de grandeur de l'influence de la vitesse sur le bruit en des points homologues de fonctionnement a été vérifiée expérimentalement?

M. BARIO. - L'ordre de grandeur a été vérifiée expérimentalement.

$\mathrm{Au}$ départ, les gens disaient que le bruit varie en $V^{6} . \mathrm{Ce}$ n'est pas forcément exact, parce que l'on est placé devant un problème de conception. On a vu que la force tangentielle $D_{R}$ n'avait pas de contribution sur le bruit, quelle que soit la vitesse de rotation.

Etant donné que les paramètres $\mathrm{T}$ et $\mathrm{D}$ ne varient pas de la même façon, il est difficile de prévoir le bruit.

M. le Président. - L'hypothèse que vous avez faite de minimiser les vitesses relatives paraît être rajoutée aux autres hypothèses qui se déduisent logiquement des formules de Lowson.

M. BARIO. - En fait, on a peu d'informations sur la contribution du rotor et du stator.

En règle générale on pense que c'est le rotor qui provoque le bruit dans une machine.

Etant donné qu'il y avait peu d'informations à ce sujet, on est parti de l'hypothèse suivante, à savoir que le bruit est une fonction de la vitesse. C'est pourquoi on a accepté de minimiser les vitesses maximales. C'est une hypothèse qui est discutable, mais de bon sens, je pense.

M. le Président. - N'a-t-on pas cherché à minimiser les facteurs de diffusion dans les aubages.

M. BARIO. - Les facteurs de diffusion équivalents (6) ont été optimisés. 


\section{Abstract}

A possibility of taking acoustic criteria into consideration in the aerodynamic calculation for an axial fan

The use of axial flow fan is more suitable than that of centrifugal blower, as soon as large mass flow with low pressure rise must be achieved. Nethertheless, the noise level of the first type of these machines is 10 to $20 \mathrm{~dB}$ higher at the same operation point.

The Metraflu and Neu companies have join their efforts to developp a theoretical study [1] and the build up of a quiet axial fan

The design technique is based on the numerical resolution of the fluid equilibrium equations. The obtained velocity triangles are used to define the blade shape [6].

The contribution of stationary and unstationary forces is examined. Unstationary forces are analysed with the hypothesis of a quasi-stationary aspect of the problem.
The noise component due to stationary forces can be explained by the formulations of page 616 . It can be seen that noise reduction can be obtained with a reduction of the terms $\Omega T, \Omega D$. As pressure rise is defined, the term $\Omega D$ is constant. Only the $\Omega T$ term could contribute to the noise reduction.

$$
\Omega T \sim U \Delta P_{S} \sim \Delta P_{S} \Delta P_{T} / \Delta V_{u} \quad \Delta P_{T}=\Delta P_{S}
$$

so

$$
\Omega T \sim C^{s t} / \Delta V_{u}
$$

The noise reduction could be obtained if important deflections are imposed (associated with low rotation speed).

The noise level of a new fan built with this concept is $5 \mathrm{~dB}$ lower than that of a reference fan known to be already quiet. 